\title{
Short-term Cardiovascular Complications of Multi-system Inflammatory Syndrome in Children (MIS-C) in Adolescents and Children
}

\author{
Omar I. Hejazi ${ }^{1} \cdot$ Yue-Hin Loke ${ }^{2,3} \cdot$ Ashraf S. Harahsheh ${ }^{2,3}[$ \\ Accepted: 29 September 2021 / Published online: 22 October 2021 \\ (c) The Author(s), under exclusive licence to Springer Science+Business Media, LLC, part of Springer Nature 2021
}

\begin{abstract}
Purpose of Review We provide the readers with a review of cardiac complications in children with multi-system inflammatory syndrome in children (MIS-C) and its short-term outcomes.

Recent Findings Recent reports described the acute cardiac manifestations of MIS-C in children and provided a glimpse of the short-term outcomes.

Summary Children with MIS-C have been reported to acutely have variable degrees of cardiac findings including abnormal cardiac enzymes, abnormal electrocardiographs, decreased systolic function, coronary artery abnormalities from coronary dilation to giant aneurysms, mitral valve regurgitation, tricuspid valve regurgitation, aortic valve insufficiency, pericardial effusion, diastolic dysfunction, abnormal cardiac strain, and abnormal cardiac MRI. The majority of these abnormalities resolved during short-term follow-up. Further studies are needed to assess if transient or persistent cardiac complications are associated with long-term adverse cardiac events in children with MIS-C.
\end{abstract}

Keywords Myocarditis · Coronary artery aneurysm · Pediatric multisystem inflammatory syndrome temporally associated with COVID-19 (PMIS) · Multi-system inflammatory syndrome in children (MIS-C) · 2019 coronavirus disease (COVID19) pandemic $\cdot$ Pediatric cardiology

\section{Introduction}

On 4/26/2020, a new illness emerged in children exposed to the severe acute respiratory syndrome coronavirus 2 (SARSCoV-2) [1]. This illness, categorized by severe inflammatory response, received multiple nomenclature including the multi-system inflammatory syndrome in children (MIS-C)

Ashraf S. Harahsheh

aharahsh@childrensnational.org

Omar I. Hejazi

omar.hejazi99@gmail.com

Yue-Hin Loke

YLoke@childrensnational.org

1 School of Medicine, University of Jordan, Amman, Jordan

2 Division of Cardiology, Children's National Hospital, 111 Michigan Ave, NW, Washington, DC 20010, USA

3 Department of Pediatrics, George Washington University School of Medicine \& Health Sciences, Washington, DC 20052, USA
$[2 \bullet, 3 \bullet]$. As we passed the first anniversary, we provide the readers with a review of cardiac complications in children with MIS-C and its short-term outcomes.

Early in the 2019 coronavirus disease (COVID-19) pandemic, previously healthy children were thought to have been spared from severe disease [4]. However, we quickly learned how unpredictable this illness can be as MIS-C emerged with reports of mortalities in the pediatric population $[5-7,8 \bullet, 9,10]$. Following early experience, centers and societies developed diagnostic and treatment algorithms to properly treat children with MIS-C $[11 \bullet \bullet, 12 \bullet \bullet]$. It is now considered prudent for providers to identify cases of MIS-C early in their illness and provide timely immune modulation therapy. Children with MIS-C have been reported to have variable degrees of cardiac findings including abnormalities of cardiac enzymes, electrocardiogram (EKG), echocardiogram, and cardiac Magnetic Resonance Imaging (MRI) (Fig. 1, Tables 1-2). 
Fig. 1 Distribution of cardiac complications in children with multi-system inflammatory syndrome in children (MIS-C). AI, aortic insufficiency; AV, atrioventricular; BNP, B-type natriuretic peptide; FS, shortening fraction; ECG, electrocardiographic; EF, ejection fraction; MR, mitral regurgitation; NT-pro BNP, N-terminal $\mathrm{B}$-type natriuretic peptide; TR, tricuspid regurgitation

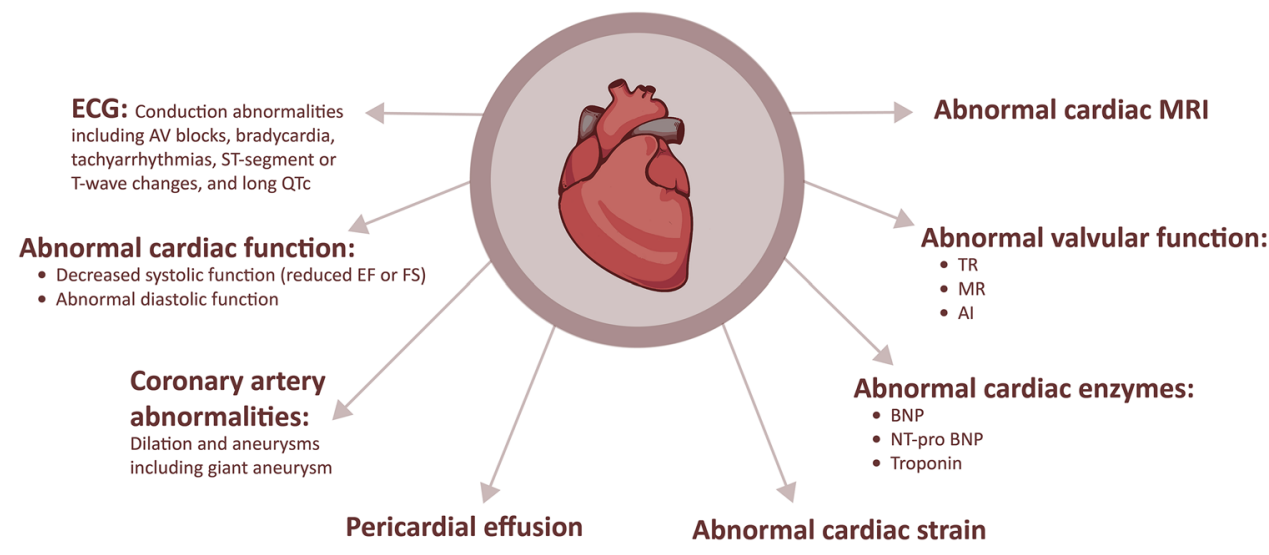

\section{Abnormal Cardiac Enzymes}

Cardiac enzymes including B-type natriuretic peptide (BNP) (pg/mL), N-terminal BNP (NT-pro BNP) (pg/mL), and troponins were noted to be elevated in some patients with MIS-C. In patients who developed shock, the median (interquartile (IQR)) troponin (ng/L) was 45 (8-294) and the median NT-pro BNP was $788(174-10,548)$ [8•]. Other reports have also described similar elevation in cardiac enzymes [13••, 14-26]. Cardiac enzyme elevation has also been reported in other cardiac diseases. Troponin (ng/L) was noted to be elevated in children with acute myocarditis 80 (10-4930) [27]. NT-pro BNP (pg/mL) was shown to be elevated in children with cardiac disease (median: 548, range: $5-35,000$ ) and helped differentiate them from other etiologies [28]. In some articles discussing MIS-C, the elevated cardiac enzymes were further stratified by clinical presentation. For example, the NT-pro BNP $(\mathrm{ng} / \mathrm{mL})$ and troponin were $2,148 \pm 259$ and $0.4 \pm 0.5$ in Kawasaki disease (KD), $7443 \pm 15,975$ and $1.0 \pm 2.0$ in incomplete KD, and 17,678 $\pm 39,609$ and 1.0 \pm 1.7 in shock presentations, respectively [29]. Matsubara et al. noted that median BNP of $596(310-1,007)$ and median troponin I $(\mathrm{ng} / \mathrm{mL})$ (nor$\mathrm{mal}<0.3)$ of $0.44(0.09-1.46)$ correlated with the global longitudinal strain (GLS), global circumferential strain rate (GCSR), left atrial strain (LAS), and the right ventricular free wall longitudinal strain (RVFWLS) [30•].

\section{Electrocardiographic Findings of MIS-C}

MIS-C causes a variety of EKG changes. Conduction abnormalities including atrioventricular (AV) blocks, bradycardia, and tachyarrhythmias including ventricular arrhythmia, ST-segment or T-wave changes, and long QTc are all possible findings in MIS-C [8•, 16, 18, 20, 26, 29, 31] (Table 2). Further studies are needed to assess correlation of ECG changes with clinical outcomes or other cardiac findings.

\section{Conventional Echocardiographic Findings of MIS-C}

Multiple echocardiographic findings have been reported in children with MIS-C including decreased systolic function, coronary artery abnormalities, valve regurgitation, and pericardial effusion.

\section{Decreased Systolic Function}

Decreased systolic function was defined as left ventricular ejection fraction (LVEF) below 55\% [32]. Decreased LVEF

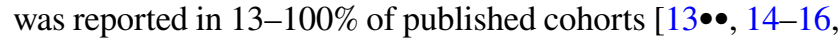
19, 21, 22, 25, 29, 30•, 33••] (Table 2). In some MIS-C patients, LV systolic dysfunction was reported in those who developed shock [8•]. LV systolic dysfunction was not only reported on admission but also occurred later during admission emphasizing the importance of frequent echocardiogram assessment in this population [26]. In a study that recruited MIS-C children with decreased LV function, 25 (71\%) had mild to moderate systolic dysfunction (an EF between 30 and 50\%) and $10(29 \%)$ patients had severe systolic dysfunction (EF below 30\%) [31]. Clark et al. further categorized the degree of systolic function impairment with $11(32 \%)$ patients with mild systolic dysfunction (EF $41-50 \%)$, and six (17\%) patients with moderate to severe systolic dysfunction (an EF < 40\%) [20]. One patient was reported by Kappanayil et al. to have a severely reduced EF of $10 \%$ [24]. What is lacking in these studies is the timing of echocardiogram to immune therapy and declaration of simultaneous inotropic or respiratory support at the time of obtaining the echocardiogram. As EF is known to be preload dependent, one wonders if frequency of systolic dysfunction 


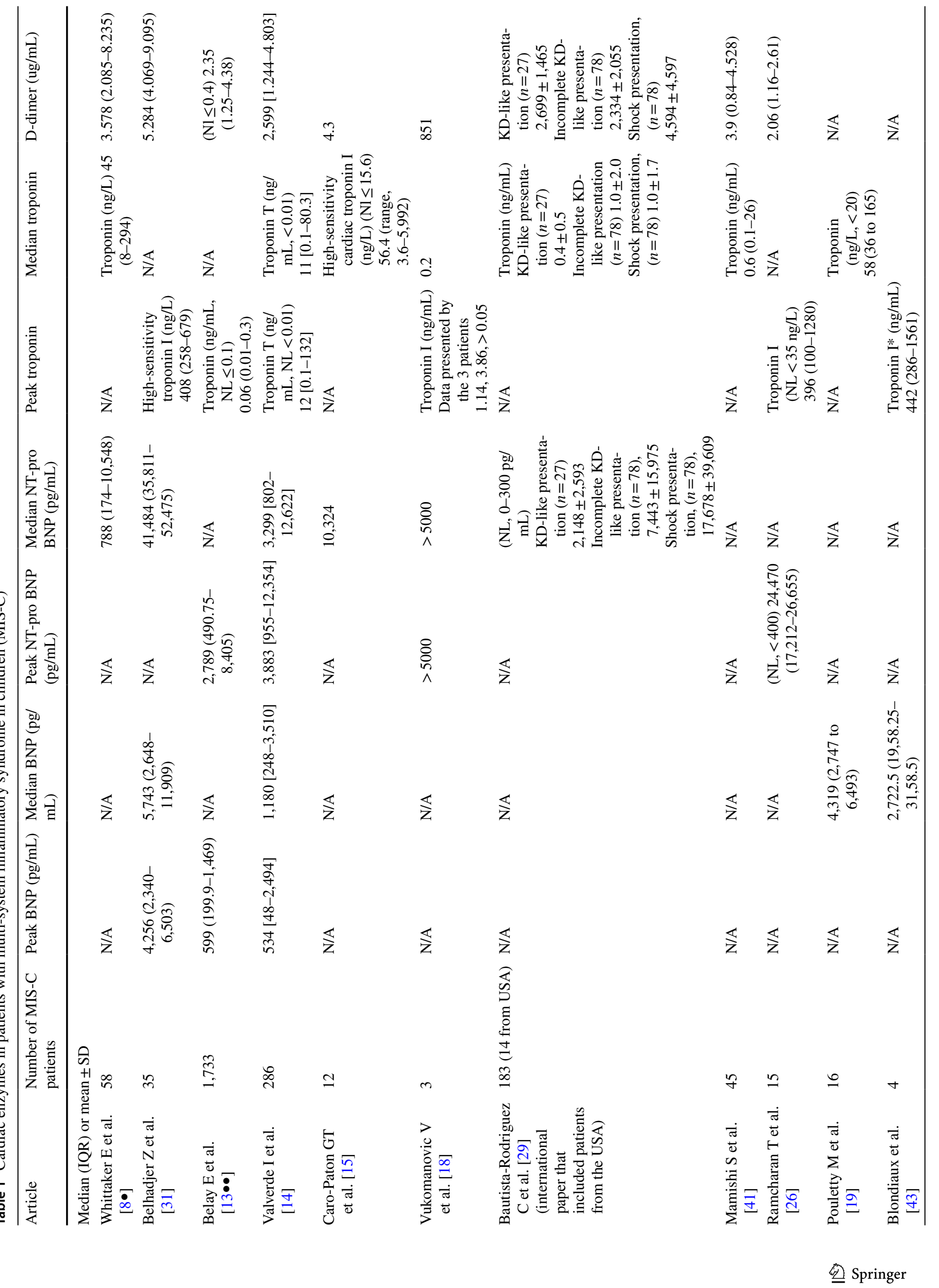




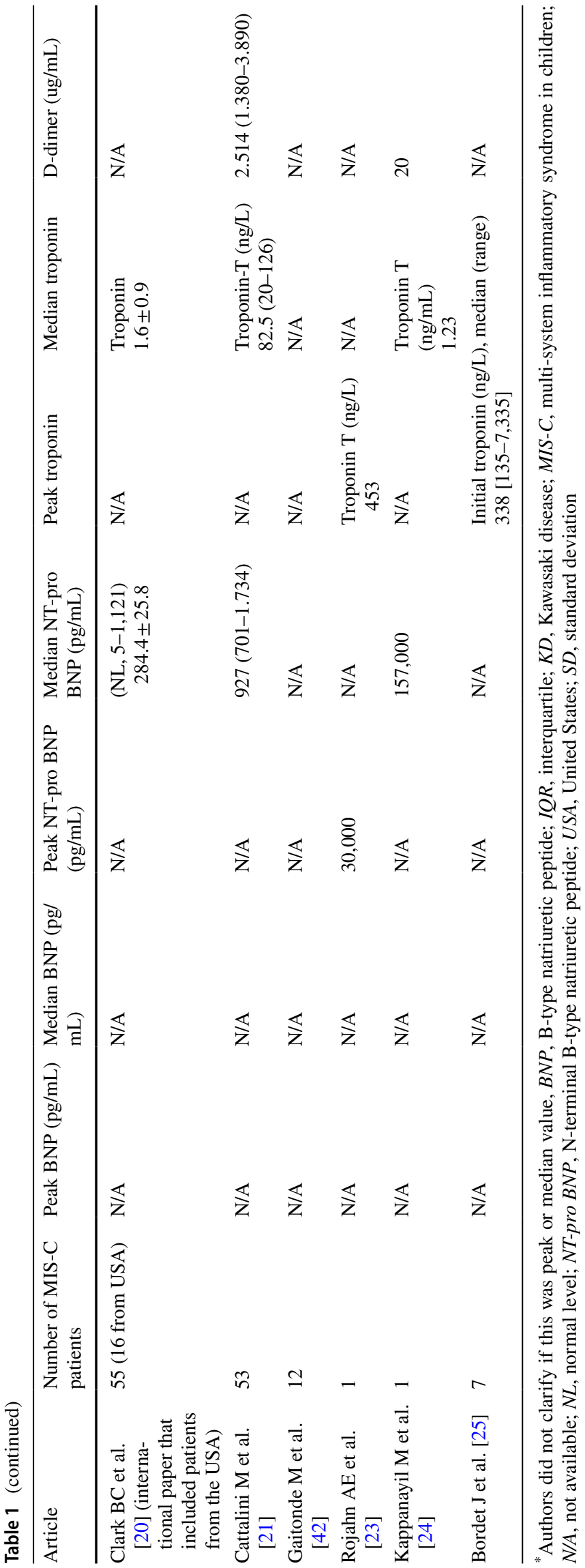

was underestimated as most studies relied on the conventional EF [34••].

\section{Coronary Artery Abnormalities}

Coronary artery abnormality (CAA) was defined as a dilation if the $Z$ score was 2 to $<2.5$, small aneurysm 2.5 to $<5$, moderate aneurysm 5 to $<10$, and giant aneurysm referred to a $Z$ score $\geq 10$ or an actual measurement of $\geq 8 \mathrm{~mm}$ and has been described in children with MIS-C raising initial interest among the KD expert community [35••, 36-39, $40 \bullet \bullet$. The literature, consisting of single case reports to cohorts of up to 1733 patients, reported CAAs in 7 to $66 \%$ of children with MIS-C in the acute phase with variable CA involvement [13••, 15-21, 26, 29, 30•, 31, 41, 42] (Table 2). When excluding studies that included less than 50 patients, the reported incidence is $14-28 \%$. While the majority of CAAs were either dilation or small aneurysms, some have reported cases of patients who developed giant aneurysms [8•]. Multivessel CAAs have also been reported $[14,33 \bullet \bullet]$.

\section{Valvular Regurgitation}

Variable degrees of atrioventricular valve regurgitation and aortic valve insufficiency (AI) have been described in children with MIS-C. When excluding studies that included less than 50 patients, the reported incidence of valvular regurgitation or insufficiency is $24-48 \%[16,17,20-22,25,26,29$, 30•, 42] (Table 2). On admission, Valverde et al. had 103 (38\%) patients with mild mitral valve regurgitation (MR), 11 (4.2\%) with moderate MR, and a single patient $(0.3 \%)$ with severe MR. On the other hand, 10 (3.8\%) patients had mild tricuspid valve regurgitation (TR) and six (2.1\%) had moderate TR [14]. It is yet to be determined if valve regurgitation in children with MIS-C relates to inflammation of the valve itself (valvulitis) or secondary to ventricular dilation. In one report, the authors concluded that the MR seen in $2(50 \%)$ patients was related to LV dilation [26].

\section{Pericardial Effusion}

Pericardial effusion occurred in $9-28 \%$ after excluding studies that included less than 50 patients (Table 2) [13••,

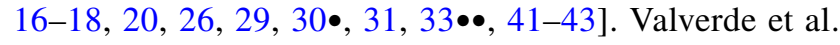
described $80(27.9 \%)$ patients to have a pericardial effusion upon admission to the hospital. While most papers did not include the degree of pericardial effusion and if it was hemodynamically significant, this study reported that 66 (23\%) were mild, 8 (3\%) were moderate, and none was severe effusions. At follow-up, pericardial effusion persisted in 59 (20.6\%) patients [14]. 


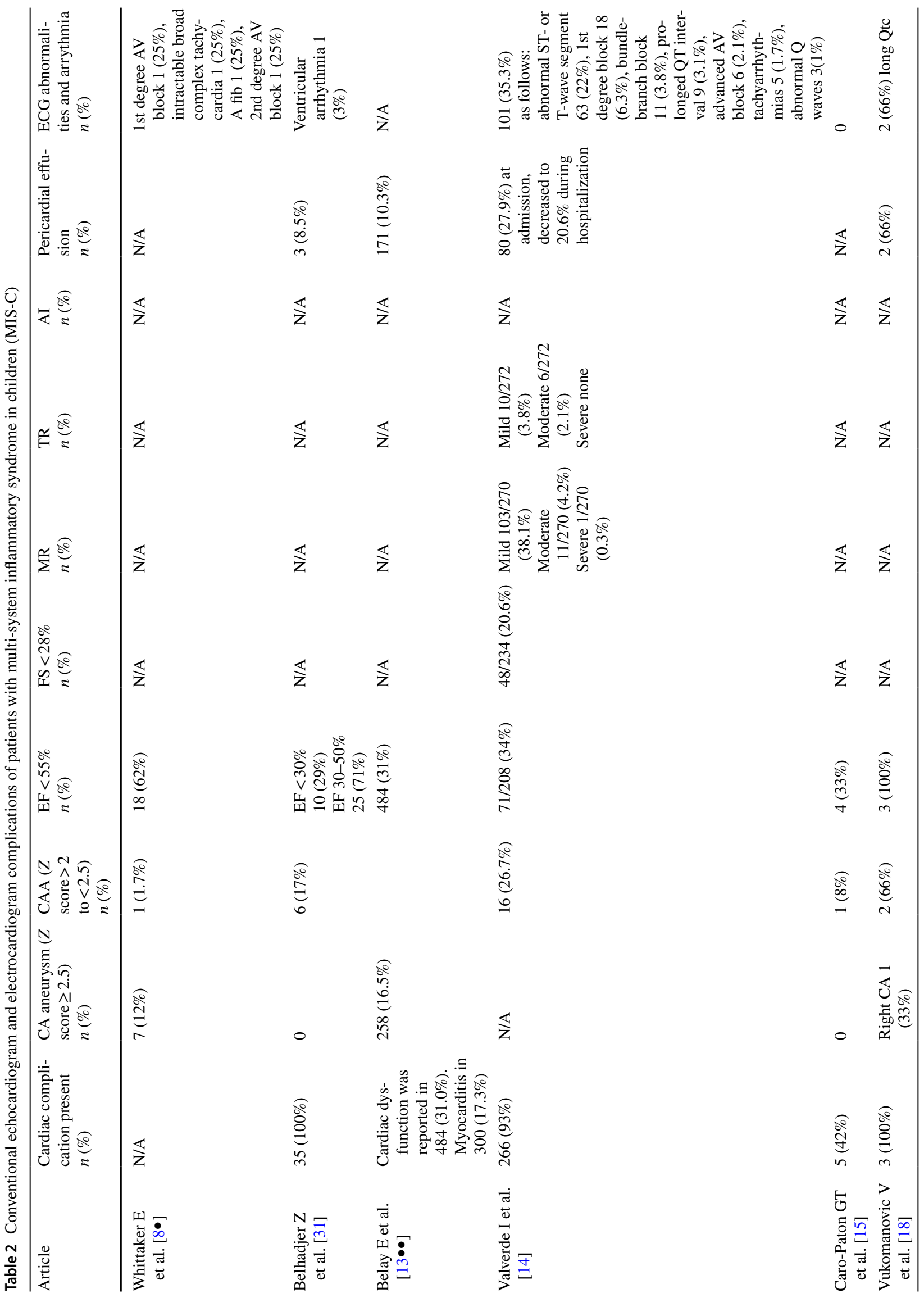




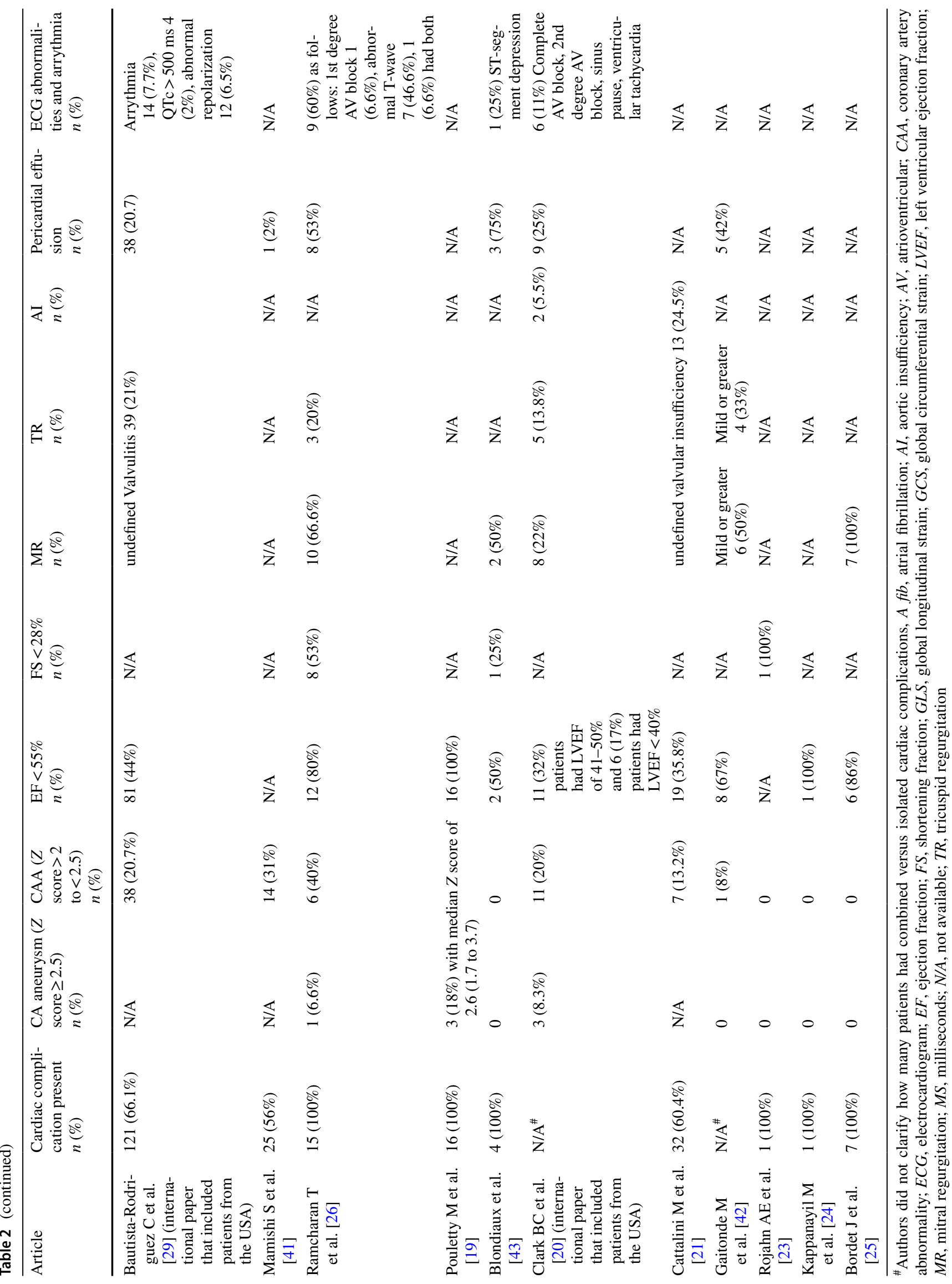




\section{Advanced Cardiac Imaging Findings of MIS-C}

\section{Cardiac Magnetic Resonance Imaging (CMR)}

In light of these clinical cardiac complications from MIS-C, multiple centers are proactively utilizing CMR to follow up these patients in prospective observational studies [44-46]. Such efforts are critical, as CMR findings from published case series are generally limited by inconsistent timing of CMR in relation to onset of disease (in some centers, CMR was performed during acute phase, whereas others waited at least 6 weeks to 3 months). In general, CMR findings include pericardial effusion, abnormal T2 myocardial values/ hyperintense myocardium on T2-weighted imaging (representing myocardial edema), early gadolinium enhancement (representing capillary leak/hyperemia), and late gadolinium enhancement (representing myocardial necrosis/fibrosis) [47]. For example, Valverde et al. reported CMR results in 42/286 (14.7\%) patients; T2 hyperintensity was found in 14 (33.3\%), pericardial effusion in $10(23.8 \%)$, early gadolinium enhancement in $1(2.4 \%)$, and late gadolinium enhancement in $6(14.3 \%)$ [14]. This is of importance as late gadolinium enhancement was found to correlate with worse composite outcome of all-cause mortality, cardiac mortality, and/ or major adverse cardiovascular events in adults with acute myocarditis [48]. Although pediatric data is very limited, late gadolinium enhancement correlated with increased risk of worse outcomes $[49,50]$. There is also ongoing assessment of native $\mathrm{T} 1 / \mathrm{T} 2$ changes by parametric mapping which are now encouraged in CMR evaluation of nonischemic myocardial inflammation $[47,51]$.

\section{Abnormal Myocardial Strain and Abnormal Diastolic Function}

Abnormal myocardial strain and abnormal diastolic function have been described in children with MIS-C. Myocardial strain takes into account the heterogenous motion of the myocardial wall, providing an angle-independent quantification of deformation in radial, circumferential, and longitudinal directions. In general, the more negative a strain value, the better it corresponds to myocardial function. However, interpreting the significance of myocardial strain across MIS-C studies is challenged by interobserver variability that is vendor dependent (machine used for acquisition, and software used for strain analysis) [52]. Reference ranges have been developed for left ventricular strain values based on age and vendor [53], and Valverde et al. used this to identify that 5/44 (11.4\%) had reduced LVGLS[14].

Other studies have opted to compare MIS-C patients against other historical cohorts such as healthy controls or KD patients. For example, Matsubara et al. reported that the median LVGLS in MISC was worse at - 16.2 (IQR - 19.5 to - 13.6), compared to healthy controls at -22.5 (IQR -23.7 to $-21.1, p<0.001)$ and classic KD at -20.1 $(-21.8$ to -18.1$)$. Other systolic strain parameters such as global circumferential strain (GCS), LAS, and RVFWLS also demonstrated similar patterns. Interestingly, diastolic parameters such as circumferential/longitudinal strain rate in early diastole and peak pulsed wave tissue Doppler diastolic velocities in lateral mitral annulus were different and persisted despite subsequent improvement in systolic function $[30 \bullet, 54,55]$.

Despite the potential variability in measurements, MIS-C studies have demonstrated the potential value of strain in determining clinical course. Matsubara et al. demonstrated that worse LVGLS, GCS, LAS, and RVFWLS were associated with clinical myocardial injury (elevated serum biomarkers) [30•]. Both Sanil et al. and Kobayashi et al. reported that patients with worse LVGLS had higher serum biomarkers and disease severity, i.e., more likely to present in shock, require intensive care unit (ICU) stay/inotropic support and mechanical life support, and longer hospital length of stay $[55,56 \bullet \bullet$. Early strain quantification may identify a sub-group to intensify monitoring and therapy to help prevent clinical deterioration.

\section{Progression of Cardiac Complications of MIS-C}

As we commemorated the 1-year anniversary of the first report of MIS-C, several publications have reported the short-term outcomes of cardiac complications in children with MIS-C. Although rare, mortality occurred in 1-2\%

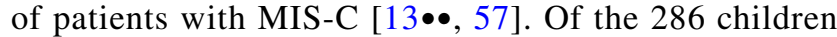
with MIS-C, one patient died after developing ventricular arrhythmia and another patient developed dilated cardiomyopathy and was listed for heart transplantation [14]. In Bautista-Rodriguez et al.'s report, a patient died after suffering a massive myocardial infarction after developing giant coronary artery aneurysms in all three vessels, another patient died while on ECMO due to cerebral injury, and a third patient died after several cardiac arrests [29].

ECG abnormalities normalized prior to discharge in $22-100 \%$ of patients at a median of 5 days in some patients $[14,20,26]$. LV dysfunction resolved in $58-100 \%$ of those with initial systolic dysfunction at a median time of only 2 days in some cohorts [14, 17, 18, 24, 31, 58]. Others also reported that all cardiac findings, including any ECG abnormalities (including 3rd degree AV block), reduced LVEF, $\mathrm{MR}$, and CAAs resolved within 2 weeks $[16,18,20]$. In Feldstein et al.'s article, during hospitalization, 172 patients (34.2\%) had LV systolic dysfunction. Most patients (91.0\% 
of cohort) normalized their LVEF by 30 days, and $99.4 \%$ of cohort had normal LVEF by 90 days. The last patient had a normal LVEF at 142 days [57].

In some cohorts, cardiac complications persisted at time of last follow-up [21, 33••]. One study found that, at the time of discharge, cardiac complications persisted in $3(25 \%)$ patients [15]. One patient had persistent LAD prominence [17]. As mentioned earlier, GLS remained low and the RV dysfunction persisted despite normalization of LVEF [30•]. Follow-up for patients in Ramcharan et al. revealed that atrioventricular valve regurgitation persisted in 7/13 (54\%) patients. The patient with the lowest EF (28\%) (severely reduced) improved to $53 \%$ (mildly reduced) at discharge. Mildly decreased systolic function persisted in 1/8 (12.5\%) patients [26]. Some studies provided follow-up data on the patients with worst disease (i.e., admitted to ICU). Only two patients out of the 7 who were admitted to the ICU had persistent mild LV dysfunction [19].

CAAs persisted in few patients with MIS-C. Although cardiac systolic dysfunction, MR, and AI resolved, mild CA enlargement and a small aneurysm in the right CA persisted in one patient [22]. In Gaitonde et al., 2 (16\%) had persistent CA dilation at time of last follow-up [42]. Sanil et al. reported resolution of CAAs in 5/6 patients at the 3-and 10 -week follow-up visits. The only patient with persistent CAA had CA $Z$ score of 2.4 (dilation) [56••]. In a study that focused on follow-up of patients with MISC, after 6 months, one patient had a medium CA aneurysm ( $Z$ score 9.8$)$ that was stable [58]. These reports highlight that although mortality did occur in children with MIS-C, it is very rare. More importantly, during the short-term follow-up, most of the cardiac findings resolved.

\section{Demographics, Clinical Features, Laboratory Evaluation, and Clinical Outcome of Children With MIS-C}

Supplemental tables 3 to 7 display the demographics, clinical presentations, laboratory evaluation therapies received, clinical course, and outcomes of children with MIS-C. To help ensure we did not double count in the tables, we excluded any US-based study that included patients prior to $1 / 11 / 2021$ as they were included in Belay et al.'s manuscript [13••] (supplemental Fig. 2).

\section{Future Direction}

As our community expands its investigations of this relatively new illness, we ought to work collaboratively to answer:
1- Why do some children with MIS-C develop cardiac complications? What are the demographics, clinical, laboratory, and therapeutic items associated with developing cardiac complications? In a small single center observational cohort study, children with cardiac complications were more likely to present with conjunctival injection, have higher NT-pro BNP, higher white blood cell count, higher neutrophil count, severe lymphopenia, use of milrinone, and intensive care requirement [33••]. Can artificial intelligence be utilized to predict which patients are at increased risk for developing cardiac abnormalities? Is there a learning curve in managing children with MIS-C? If so, is it associated with development of cardiac complications? Case in point, patients seen after 7/1/2020 were more likely to receive immune therapy and less likely to develop cardiac complications compared to those evaluated prior to $07 / 01 / 2020$ [13••].

2- What are the factors favoring resolution of cardiac findings in children with MIS-C? What is the role of immune therapy choice and/or timing in resolving the cardiac findings? Is there a "Golden hour" for initiating therapy? [7].

3- What are the long-term cardiac findings in children with MIS-C? Are the cardiac changes seen in the acute phase, transiently or persistently, associated with long-term cardiac abnormalities including cardiomyopathy?

\section{Conclusions}

Cardiac complications in children with MIS-C are not uncommon and have been reported across the globe. Although mortality has occurred, it is very rare and the majority of children with MIS-C had resolution of their cardiac complications. Further studies are needed to assess if transient or persistent cardiac complications are associated with long-term adverse cardiac events.

Supplementary Information The online version contains supplementary material available at https://doi.org/10.1007/s40124-021-00258-5.

Acknowledgements The authors wish to thank Angela J. Doty, MD, and Lindsay Attaway for their editorial assistance.

Author Contribution Omar I. Hejazi contributed to literature review and drafting the manuscript. Yue-Hin Loke was involved in literature review and with revising the manuscript. Ashraf S. Harahsheh was involved in manuscript design, literature review, and manuscript revision. All authors approved the final manuscript as submitted and agree to be accountable for all aspects of the work.

Funding All authors have reported that they have no relationships relevant to the contents of this paper to disclose. This research did not receive any specific grant from funding agencies in the public, commercial, or not-for-profit sectors. Dr. Harahsheh is supported by 
a sub-agreement from the Johns Hopkins University with funds provided by Grant No. R61HD105591 from the Eunice Kennedy Shriver National Institute of Child Health \& Human Development and the Office of the Director, National Institute of Health (OD). Dr. Loke receives partial salary support from the National Heart, Lung, and Blood Institute (NHLBI)-National Institute of Health Grant No. R21 HL156045-01 and the National Institute of Biomedical Imaging and Bioengineering (NIBIB) Grant No. R01 HL143468-01. Its contents are solely the responsibility of the authors and do not necessarily represent the official views of the Eunice Kennedy Shriver National Institute of Child Health \& Human Development, the Office of the Director, National Institute of Health (OD), the National Institute of Health, the NIBIB, the NHLBI, or the Johns Hopkins University.

Availability of Data and Material Data will be available upon a reasonable request.

\section{Declarations}

Conflict of Interest The authors declare no competing interests.

Human and Animal Rights and Informed Consent All reported studies/ experiments with human or animal subjects performed by the authors have been previously published and complied with all applicable ethical standards (including the Helsinki declaration and its amendments, institutional/national research committee standards, and international/ national/institutional guidelines).

Clinical Trial Registration Not applicable.

\section{References}

Papers of particular interest, published recently, have been highlighted as:

- Of importance

$\bullet$ Of major importance

1. Paediatric multisystem inflammatory syndrome temporally associated with COVID-19. https://www.rcpch.ac.uk/sites/default/ files/2020-05/COVID-19-Paediatricmultisystem-\%20inflamma tory\%20syndrome-20200501.pd. Accessed on 5/10/2020.

2. Multisystem inflammatory syndrome in children (MIS-C) associated with coronavirus disease 2019 (COVID-19) https://emerg ency.cdc.gov/han/2020/han00432.asp. Accessed on 5/16/2020. The CDC case definition utilized in the United States.

3. Loke YH, Berul CI, Harahsheh AS. Multisystem inflammatory syndrome in children: is there a linkage to Kawasaki disease? Trends Cardiovasc Med. 2020;30(7):389-396. A review article discussing similarities and differences between MIS-C and Kawasaki disease.

4. DeBiasi RL, Song X, Delaney M, et al. Severe coronavirus disease-2019 in children and young adults in the Washington, DC, Metropolitan Region. J Pediatr. 2020;223:199-203 e191.

5. Harahsheh AS, Selekman RE, Simpson JN, et al. Children's hospital ambulatory response to the 2019 novel coronavirus disease (COVID-19) pandemic. J Ambul Care Manage. 2021.

6. Harahsheh AS, Dahdah N, Newburger JW, et al. Missed or delayed diagnosis of Kawasaki disease during the 2019 novel coronavirus disease (COVID-19) pandemic. J Pediatr. 2020;222:261-2.
7. Mahmoud S, Fouda EM, Kotby A, et al. The "golden hours" algorithm for the management of the multisystem inflammatory syndrome in children (MIS-C). Glob Pediatr Health. 2021;8:2333794X21990339.

8. Whittaker E, Bamford A, Kenny J, et al. Clinical characteristics of 58 children with a pediatric inflammatory multisystem syndrome temporally associated with SARS-CoV-2. JAMA. 2020;324(3):259-269. Landmark paper comparing MIS-C to historical Kawasaki disease.

9. Harahsheh AS, Dahdah N, Newburger JW, et al. Reply. J Pediatr. 2020;224:184-185.e1.

10. Abdel-Haq N, Asmar BI, Deza Leon MP, et al. SARS-CoV2-associated multisystem inflammatory syndrome in children: clinical manifestations and the role of infliximab treatment. Eur J Pediatr. 2021;180(5):1581-91.

11.• DeBiasi RL, Harahsheh AS, Srinivasalu H, et al. Multisystem inflammatory syndrome of children: sub-phenotypes, risk factors, biomarkers, cytokine profiles and viral sequencing. J Pediatr. 2021;237:125-135.e18. A prospective case control study comparing MIS-C sub-phenotypes to controls.

12.• Elias MD, McCrindle BW, Larios G, et al. Management of multisystem inflammatory syndrome in children associated with COVID-19: a survey from the International Kawasaki Disease Registry. CJC Open. 2020;2(6):632-640. International survey describing therapeutic strategies in children with MIS-C.

13.• Belay ED, Abrams J, Oster ME, et al. Trends in geographic and temporal distribution of US children with multisystem inflammatory syndrome during the COVID-19 pandemic. JAMA Pediatr. 2021 Aug 1;175(8):837-45. One of the largest US-based MIS-C cohort comparing early to late onset cases.

14. Valverde I, Singh Y, Sanchez-de-Toledo J, et al. Acute cardiovascular manifestations in 286 children with multisystem inflammatory syndrome associated with COVID-19 infection in Europe. Circulation. 2021;143(1):21-32.

15. Caro-Paton GL, de Azagra-Garde AM, Garcia-Salido A, Cabrero-Hernandez M, Tamariz A, Nieto-Moro M. Shock and myocardial injury in children with multisystem inflammatory syndrome associated with SARS-CoV-2 infection: what we know. Case Series and Review of the Literature. J Intensive Care Med. 2021;36(4):392-403.

16. Minocha PK, Phoon CKL, Verma S, Singh RK. Cardiac findings in pediatric patients with multisystem inflammatory syndrome in children associated with COVID-19. Clin Pediatr (Phila). 2021;60(2):119-26.

17. Jain S, Nolan SM, Singh AR, et al. Myocarditis in multisystem inflammatory syndrome in children associated with coronavirus disease 2019. Cardiol Rev. 2020;28(6):308-11.

18. Vukomanovic V, Krasic S, Minic P, et al. Kawasaki-like disease and acute myocarditis in the SARS-CoV-2 pandemic-reports of three adolescents. Bosn J Basic Med Sci. 2021;21(2):252.

19. Pouletty M, Borocco C, Ouldali N, et al. Paediatric multisystem inflammatory syndrome temporally associated with SARSCoV-2 mimicking Kawasaki disease (Kawa-COVID-19): a multicentre cohort. Ann Rheum Dis. 2020;79(8):999-1006.

20. Clark BC, Sanchez-de-Toledo J, Bautista-Rodriguez C, et al. Cardiac abnormalities seen in pediatric patients during the SARS-CoV2 pandemic: an international experience. J Am Heart Assoc. 2020;9(21):e018007.

21. Cattalini M, Della Paolera S, Zunica F, et al. Defining Kawasaki disease and pediatric inflammatory multisystem syndrometemporally associated to SARS-CoV-2 infection during SARSCoV-2 epidemic in Italy: results from a national, multicenter survey. Pediatr Rheumatol Online J. 2021;19(1):29.

22. Regev T, Antebi M, Eytan D, et al. Pediatric inflammatory multisystem syndrome with central nervous system involvement and 
hypocomplementemia following SARS-COV-2 infection. Pediatr Infect Dis J. 2020;39(8):e206-7.

23. Rojahn AE, Gammelsrud KW, Brunvand LI, et al. Multiorgan inflammatory syndrome associated with SARS-CoV-2 in a child. Tidsskr Nor Laegeforen. 2020;140(11).

24. Kappanayil M, Balan S, Alawani S, et al. Multisystem inflammatory syndrome in a neonate, temporally associated with prenatal exposure to SARS-CoV-2: a case report. Lancet Child Adolesc Health. 2021;5(4):304-8.

25. Bordet J, Perrier S, Olexa C, Gerout AC, Billaud P, Bonnemains L. Paediatric multisystem inflammatory syndrome associated with COVID-19: filling the gap between myocarditis and Kawasaki? Eur J Pediatr. 2021;180(3):877-84.

26. Ramcharan T, Nolan O, Lai CY, et al. Paediatric inflammatory multisystem syndrome: temporally associated with SARS-CoV-2 (PIMS-TS): cardiac features, management and short-term outcomes at a UK tertiary paediatric hospital. Pediatr Cardiol. 2020;41(7):1391-401.

27. Soongswang J, Durongpisitkul K, Nana A, et al. Cardiac troponin T: a marker in the diagnosis of acute myocarditis in children. Pediatr Cardiol. 2005;26(1):45-9.

28. Kim HS, Choi HJ. N-terminal pro-B-type natriuretic peptide levels in children: comparison in cardiac and non-cardiac diseases. Cardiol Young. 2020;30(4):500-4.

29. Bautista-Rodriguez C, Sanchez-de-Toledo J, Clark BC, et al. Multisystem inflammatory syndrome in children: an international survey. Pediatrics. 2021;147(2).

30. Matsubara D, Kauffman HL, Wang Y, et al. Echocardiographic findings in pediatric multisystem inflammatory syndrome associated with COVID-19 in the United States. J Am Coll Cardiol. 2020;76(17):1947-1961. Single center study comparing MIS-C to Kawasaki disease and controls.

31. Belhadjer Z, Meot M, Bajolle F, et al. Acute heart failure in multisystem inflammatory syndrome in children in the context of global SARS-CoV-2 pandemic. Circulation. 2020;142(5):429-36.

32. Margossian R, Schwartz ML, Prakash A, et al. Comparison of echocardiographic and cardiac magnetic resonance imaging measurements of functional single ventricular volumes, mass, and ejection fraction (from the Pediatric Heart Network Fontan Cross-Sectional Study). Am J Cardiol. 2009;104(3):419-28.

33.• Harahsheh AS, Krishnan A, DeBiasi RL, et al. Cardiac echocardiogram findings of severe acute respiratory syndrome coronavirus-2-associated multi-system inflammatory syndrome in children. Cardiol Young. 2021;5:1-9. https://doi.org/10.1017/ S1047951121003024. Single center study assessing risk factors associated with cardiac complications.

34.•• Lopez L, Colan SD, Frommelt PC, et al. Recommendations for quantification methods during the performance of a pediatric echocardiogram: a report from the Pediatric Measurements Writing Group of the American Society of Echocardiography Pediatric and Congenital Heart Disease Council. J Am Soc Echocardiogr. 2010;23(5):465-495; quiz 576-467. Excellent reference discussing echocardiogram evaluation of children.

35.• McCrindle BW, Rowley AH, Newburger JW, et al. Diagnosis, treatment, and long-term management of Kawasaki disease: a scientific statement for health professionals from the American Heart Association. Circulation. 2017;135(17):e927-e999. Excellent reference discussing management of children with Kawasaki disease.

36. Manlhiot C, Newburger JW, Low T, et al. Low-molecular-weight heparin vs warfarin for thromboprophylaxis in children with coronary artery aneurysms after Kawasaki disease: a pragmatic registry trial. Can J Cardiol. 2020;36(10):1598-607.

37. McCrindle BW, Manlhiot C, Newburger JW, et al. Medium-term complications associated with coronary artery aneurysms after
Kawasaki disease: a study from the international Kawasaki Disease Registry. J Am Heart Assoc. 2020;9(15):e016440.

38. Osborne J, Friedman K, Runeckles K, et al. Comparison between currently recommended long-term medical management of coronary artery aneurysms after Kawasaki disease and actual reported management in the last two decades. Pediatr Cardiol. 2021;42(3):676-84.

39. Teitel DF, Newburger JW, Sutton N, et al. Development and utility of quality metrics for ambulatory pediatric cardiology in Kawasaki disease. Clin Pediatr (Phila). 2020;59(3):245-51.

40.• Lamrani L, Manlhiot C, Elias MD, et al. Kawasaki disease shock syndrome versus classical Kawasaki disease, a meta-analysis and comparison with SARS-CoV-2 multisystem inflammatory syndrome. Can J Cardiol. 2021;6:S0828-282X(21)00290-7. Excellent systemic review and meta-analysis comparing Kawasaki disease to Kawasaki disease shock syndrome and MIS-C..

41. Mamishi S, Movahedi Z, Mohammadi M, et al. Multisystem inflammatory syndrome associated with SARS-CoV-2 infection in 45 children: a first report from Iran. Epidemiol Infect. 2020;148:e196.

42. Gaitonde M, Ziebell D, Kelleman MS, et al. COVID-19-related multisystem inflammatory syndrome in children affects left ventricular function and global strain compared with Kawasaki disease. J Am Soc Echocardiogr. 2020;33(10):1285-7.

43. Blondiaux E, Parisot P, Redheuil A, et al. Cardiac MRI in children with multisystem inflammatory syndrome associated with COVID-19. Radiology. 2020;297(3):E283-8.

44. Long-term outcomes after the multisystem inflammatory syndrome in children. https://covidmusicstudy.com/ Accessed on 06/21/2021.

45. COVID-19: pediatric research immune network on SARS-CoV-2 and MIS-C (PRISM). https://clinicaltrials.gov/ct2/show/NCT04 588363. Accessed on 06/21/2021.

46. Pediatric SARS-CoV-2 and MIS-C long-term follow-up. https:// clinicaltrials.gov/ct2/show/NCT04830852?term=DeBiasi\& draw $=2 \&$ rank $=1$. Accessed on 06/21/2021.

47. Ferreira VM, Schulz-Menger J, Holmvang G, et al. Cardiovascular magnetic resonance in nonischemic myocardial inflammation: expert recommendations. J Am Coll Cardiol. 2018;72(24):3158-76.

48. Georgiopoulos G, Figliozzi S, Sanguineti F, et al. Prognostic impact of late gadolinium enhancement by cardiovascular magnetic resonance in myocarditis: a systematic review and metaanalysis. Circ Cardiovasc Imaging. 2021;14(1):e011492.

49. Banka P, Robinson JD, Uppu SC, et al. Cardiovascular magnetic resonance techniques and findings in children with myocarditis: a multicenter retrospective study. J Cardiovasc Magn Reson. 2015;17:96.

50. Sachdeva S, Song X, Dham N, Heath DM, DeBiasi RL. Analysis of clinical parameters and cardiac magnetic resonance imaging as predictors of outcome in pediatric myocarditis. Am J Cardiol. 2015;115(4):499-504.

51. Messroghli DR, Moon JC, Ferreira VM, et al. Clinical recommendations for cardiovascular magnetic resonance mapping of $\mathrm{T} 1, \mathrm{~T} 2, \mathrm{~T} 2 *$ and extracellular volume: a consensus statement by the Society for Cardiovascular Magnetic Resonance (SCMR) endorsed by the European Association for Cardiovascular Imaging (EACVI). J Cardiovasc Magn Reson. 2017;19(1):75.

52. Farsalinos KE, Daraban AM, Unlu S, Thomas JD, Badano LP, Voigt JU. Head-to-head comparison of global longitudinal strain measurements among nine different vendors: the EACVI/ ASE inter-vendor comparison study. J Am Soc Echocardiogr. 2015;28(10):1171-1181, e1172.

53. Levy PT, Machefsky A, Sanchez AA, et al. Reference ranges of left ventricular strain measures by two-dimensional speckletracking echocardiography in children: a systematic review and meta-analysis. J Am Soc Echocardiogr. 2016;29(3):209-225 e206. 
54. Theocharis P, Wong J, Pushparajah K, et al. Multimodality cardiac evaluation in children and young adults with multisystem inflammation associated with COVID-19. Eur Heart J Cardiovasc Imaging. 2021;20;22(8):896-903.

55. Kobayashi R, Dionne A, Ferraro A, et al. Detailed assessment of left ventricular function in multisystem inflammatory syndrome in children using strain analysis. CJC Open. 2021;3(7):880-7.

56.• Sanil Y, Misra A, Safa R, et al. Echocardiographic indicators associated with adverse clinical course and cardiac sequelae in multisystem inflammatory syndrome in children with coronavirus disease 2021;34(8):862-76. J Am Soc Echocardiogr. 2021. Excellent single center paper discussing the use of cardiac strain in the evaluation of children with MIS-C.
57. Feldstein LR, Tenforde MW, Friedman KG, et al. Characteristics and outcomes of US children and adolescents with multisystem inflammatory syndrome in children (MIS-C) compared with severe acute COVID-19. JAMA. 2021;325(11):1074-87.

58. Penner J, Abdel-Mannan O, Grant K, et al. 6-month multidisciplinary follow-up and outcomes of patients with paediatric inflammatory multisystem syndrome (PIMS-TS) at a UK tertiary paediatric hospital: a retrospective cohort study. Lancet Child Adolesc Health. 2021;5(7):473-82.

Publisher's Note Springer Nature remains neutral with regard to jurisdictional claims in published maps and institutional affiliations. 